\title{
Computing Green Currents via the Heat Kernel
}

\author{
Georg Hein
}

July 4, 2000

\section{Introduction}

Let $X$ be a compact Kähler manifold with Kähler form $\omega$. For an analytic cycle $Y \subset X$ of complex codimension $k$ the Poincaré duality assigns a class in the de Rham cohomology which can be represented by a harmonic $(k, k)$-form $\mu_{Y, \infty}$. A Green current $g_{Y}$ connects both objects by the equality

$$
d d^{c} g_{Y}=\left[\mu_{Y, \infty}\right]-\delta_{Y} .
$$

Thus we can reconstruct $Y$ and its harmonic projection $\mu_{Y, \infty}$ from this equation, because $Y$ is the locus where $d d^{c} g_{Y}$ is not smooth, while $\mu_{Y, \infty}$ is determined by its values on the dense open set $X \backslash Y$. Furthermore, the above equality tells us that $\delta_{Y}$ and $\mu_{Y, \infty}$ differ only by some exact current.

Green currents for varieties of arbitrary dimension were systematically developed by H. Gillet and C. Soulé in [2]. These currents play a central role in Arakelov theory (see [8] and [6]). On the one hand the existence of Green currents follows more or less formally from the Hodge decomposition for currents. To do intersection theory, on the other hand, we need currents which behave well. Green currents of logarithmic type are an example of these currents (see $[2])$.

Here we present a method to find a canonical Green current $g_{Y}$ which depends only on the Kähler manifold $(X, \omega)$ and the cycle $Y$. This current $g_{Y}$ is given by $(k, k)$-forms with $L^{1}$ coefficients which are smooth on $X \backslash Y$.

We start with repeating some basic results on Kähler manifolds in 1 which can be found in the textbooks [4] or [9]. In section 2 we recall the main properties of the heat kernel before we consider the heat flux of Dirac currents in section 3 obtaining a Green operator for these currents. In 4 we define the canonical harmonic Green current and show that its harmonic projection is zero. Following [2] we investigate the affine space of all Green currents for a given cycle. In section 5 we apply the heat kernel to intersections of properly intersecting cycles. This is needed to derive a formula for the $*$-product of Green currents in 6 . However, we have to restrict to cycles that intersect properly. Hence our results are weaker than those of [2]. As we used a different approach, we decided to present them here. In section 7 we give examples which we found by a good guess (7.1) or because we know all eigen functions of the Laplace operator (7.2) or from the explicit form of the heat kernel (7.3). The last example shows how our techniques can be extended to non-compact Kähler manifolds for which a heat kernel exists. In section 8 we show how example 7.1 can be computed without guessing. The techniques of [5] presented here should be applicable to compute more concrete examples, e.g. canonical Green currents of (not too difficult) cycles in $\mathbb{P}^{n}$ and Schubert cycles in Grassmannians (at least Grass $(4,2))$. We conclude in section 9 with some questions and remarks.

Acknowledgment. The author wishes to thank W. Gubler, J. Kramer, and U. Kühn for many discussions which contributed to this paper. 


\section{Notations and preliminaries}

1.1 Our notations follow the book [4] of P. Griffith and J. Harris.

Let $X$ be a smooth compact Kähler manifold of complex dimension $n$. By $\omega$ we denote the Kähler form of $X$. The space $A^{k}(X)$ of smooth $k$-forms decomposes by type

$$
A^{k}(X)=\bigoplus_{p+q=k} A^{p, q}(X)
$$

Furthermore, $d$ decomposes as $d=\partial+\bar{\partial}$. We consider the Kähler twist

$$
L: A^{p, q}(X) \rightarrow A^{p+1, q+1}(X)
$$

which is defined by $L(\eta)=\eta \wedge \omega$. Its adjoint operator is denoted by $\Lambda$.

$$
\Lambda=L^{*}: A^{p, q}(X) \rightarrow A^{p-1, q-1}(X) .
$$

The adjoint operators for $\partial, \bar{\partial}$, and $d$ are denoted by $\partial^{*}, \bar{\partial}^{*}$, and $d^{*}$ respectively. We recall the following important equality for the Laplacian $\Delta$ on a Kähler manifold:

$$
\Delta=d d^{*}+d^{*} d=2\left(\partial \partial^{*}+\partial^{*} \partial\right)=2\left(\bar{\partial} \bar{\partial}^{*}+\bar{\partial}^{*} \bar{\partial}\right) .
$$

This implies that the Laplacian commutes with $\partial$ and $\bar{\partial}$. For the sequel the Hodge identity

$$
\Lambda \partial-\partial \Lambda=i \bar{\partial}^{*}
$$

will be needed.

1.2 The linear functionals on $A^{k}(X)$ which are continuous for the Schwartz topology form the space $D_{k}(X)$ of $k$-currents.

The decomposition of $A^{k}(X)$ by type induces a decomposition $D_{k}(X)=\oplus_{p+q=k} D_{p, q}(X)$.

We define

$$
D^{k}(X):=D_{2 n-k}(X) \text { and } D^{p, q}(X):=D_{n-p, n-q}(X) .
$$

There are two basic examples of currents:

1.3 The current associated to a form. For $\eta \in A^{p, q}(X)$ we define a current $[\eta] \in D^{p, q}(X)$ by

$$
[\eta](\alpha)=\int_{X} \eta \wedge \alpha
$$

Since $\eta$ is a smooth form, the current $[\eta]$ is called a smooth current. We obtain an embedding $A^{p, q}(X) \subset D^{p, q}(X)$ by assigning a smooth $(p, q)$-form its associated current. To assign to a form $\eta$ a current we can allow $(p, q)$-forms $\eta$ whose coefficients are locally integrable functions. We can wedge a current $T \in D^{k}(X)$ with a smooth form $\alpha \in A^{l}(X)$ by setting $(\alpha \wedge T)(\beta):=$ $(-1)^{k l} T(\alpha \wedge \beta)$, and $(T \wedge \alpha)(\beta):=T(\alpha \wedge \beta)$. This is compatible with the embedding of smooth forms into currents.

1.4 Let $\iota: Y \rightarrow X$ be an irreducible cycle (possibly singular) of codimension $p$. We choose a desingularization $\psi: \tilde{Y} \rightarrow Y$. The Dirac current $\delta_{Y} \in A^{p, p}(X)$ associated to $Y$ is defined by

$$
\delta_{Y}(\alpha)=\int_{\tilde{Y}} \psi^{*} \iota^{*} \alpha
$$

To compute $\delta_{Y}(\alpha)$ we just have to integrate $\iota^{*} \alpha$ over the smooth part of $Y$. The existence of a desingularization is needed to ensure the convergence of this integral. 
We can combine the Dirac currents with those of 1.3 by taking a locally integrable form $\eta$ on $Y$ and considering the current $T_{\eta}$ defined by

$$
T_{\eta}(\alpha)=\int_{\tilde{Y}} \psi^{*} \eta \wedge \psi^{*} \iota^{*} \alpha
$$

1.5 We define derivatives and formal adjoints of a current $T \in D^{p, q}(X)$ by

$$
\begin{array}{cc}
\partial T(\alpha)=-(-1)^{p+q} T(\partial \alpha) & \bar{\partial} T(\alpha)=-(-1)^{p+q} T(\bar{\partial} \alpha) \\
\partial^{*} T(\alpha)=-(-1)^{p+q} T\left(\partial^{*} \alpha\right) & \bar{\partial}^{*} T(\alpha)=-(-1)^{p+q} T\left(\bar{\partial}^{*} \alpha\right) .
\end{array}
$$

We set $d=\partial+\bar{\partial}$ and $d^{*}=\partial^{*}+\bar{\partial}^{*}$. By Stoke's theorem we obtain a commutative diagram

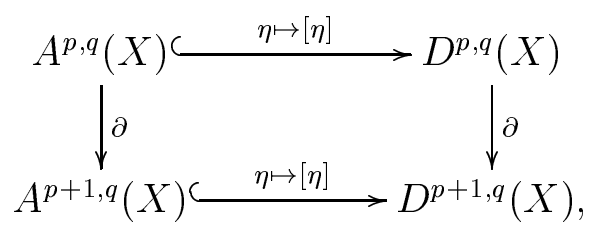

and analogously for $d, \bar{\partial}, d^{*}, \partial^{*}$ and $\bar{\partial}^{*}$. Again by Stoke's theorem, we see that $d\left(\delta_{Y}\right)=0$ for a closed cycle $Y \subset X$.

\section{The Heat Kernel on compact manifolds}

2.1 Let $X$ be a compact Kähler manifold of complex dimension $n$. As before we consider the Laplace operator $\Delta=d d^{*}+d^{*} d$ acting on the spaces $A^{k}(X)$ of smooth $k$-forms with values in $\mathbb{C}$, for $k=0, \ldots, 2 n$. The heat flux of a smooth $k$-form $\alpha$ is a family $\left\{\alpha_{t}\right\}_{t \in \mathbb{R}^{+}}$of smooth $k$-forms which are $C^{1}$ with respect to $t$ and fulfill the following two conditions:

Initial condition $\lim _{t \rightarrow 0} \alpha_{t}=\alpha$;

Evolution condition $\frac{\partial}{\partial t} \alpha_{t}=-\Delta \alpha_{t}$.

There exists a universal solution of the heat equation: the heat kernel. The next results resumes its main properties. First, let us introduce some more notations.

The projections of $X \times X$ to the first and the second component are denoted by $\mathrm{pr}_{1}$ and $\mathrm{pr}_{2}$ respectively. We denote by $\tau$ the involution automorphism of $X \times X$ which interchanges the components. For a $2 n+k$ form $\beta$ on $X \times X$ we denote by $\operatorname{pr}_{1 *} \beta$ the integration on the fibres of $\mathrm{pr}_{1}$. Thus, $\mathrm{pr}_{1 *} \beta$ is a $k$-form on $X$.

2.2 Theorem. Using the notations of 2.1 there exists a family $p_{t}$ of smooth $2 n$-forms on $X \times X$ depending smoothly on $t \in \mathbb{R}^{+}$such that:

(1) The operator $P_{t}: A^{k}(X) \rightarrow A^{k}(X)$ defined by $P_{t}(\alpha)=\operatorname{pr}_{1 *}\left(p_{t} \wedge \operatorname{pr}_{2}^{*} \alpha\right)$ "solves" the heat equation, for all $\alpha \in A^{k}(X)$, i.e., $\alpha_{t}=P_{t}(\alpha)$ is the heat flux of $\alpha$.

(2) The form $p_{t}$ is invariant under $\tau$, i.e., $\tau^{*} p_{t}=p_{t}$.

(3) For a smooth $k$-form $\alpha \in A^{k}(X)$. The limit $\alpha_{\infty}=\lim _{t \rightarrow \infty} P_{t}(\alpha)$ is the harmonic projection of $\alpha$, i.e., $\Delta \alpha_{\infty}=0$, and all integrals in the following formal computation are well defined:

$$
\alpha_{\infty}-\alpha=\int_{0}^{\infty} \frac{\partial}{\partial t} \alpha_{t} d t=\int_{0}^{\infty}-\Delta \alpha_{t} d t=-\Delta \int_{0}^{\infty}\left(\alpha_{t}-\alpha_{\infty}\right) d t
$$


For proof of the theorem see chapter 2 in [1]. Let us remark that we prefer $p_{t}$ to be a $2 n$-form. Usually it is considered to be a section in the tensor product of certain half density bundles. These density bundles allow the integration of a function on a manifold. Our point of view is that on $X$ we can integrate only $2 n$-forms.

2.3 We will need a local expression for the heat kernel near the diagonal for small $t$. Let us introduce coordinates on $X \times X$ near a point $(0,0)$ at the diagonal. We choose complex coordinates $\left\{z_{j}\right\}_{j=1, \ldots, n}$ on $X$ such that at $0 \in X$ the inner products are given by $\left\langle d z_{j}, d z_{k}>=\right.$ $2 \delta_{j k}$. We take now coordinates $\left\{z_{j}, z_{j}^{\prime}\right\}_{j=1, \ldots, n}$ on $X \times X$ such that the diagonal is given by the equations $z_{j}=z_{j}^{\prime}$, for $j=1, \ldots, n$. As usual, we decompose $z_{j}=x_{j}+i y_{j}$, and $z_{j}^{\prime}=x_{j}^{\prime}+i y_{j}^{\prime}$. For abbreviation we write $z$ instead of the $n$-tupel $\left(z_{1}, \ldots, z_{n}\right)$. As usual $\|z\|$ is defined by $\|z\|^{2}=\sum_{j=1}^{n}\left|z_{j}\right|^{2}$. Under these assumptions the heat kernel $p_{t}$ is given by

$$
p_{t}\left(z, z^{\prime}\right)=\frac{\exp \left(\frac{-\left\|z-z^{\prime}\right\|^{2}}{4 t}\right)}{(4 \pi t)^{n}} d\left(x_{1}^{\prime}-x_{1}\right) \wedge d\left(y_{1}^{\prime}-y_{1}\right) \wedge \ldots \wedge d\left(x_{n}^{\prime}-x_{n}\right) \wedge d\left(y_{n}^{\prime}-y_{n}\right)+\rho .
$$

where $\rho$ is a $2 n$-form with bounded coefficients, for $\|z\|$, and $\left\|z^{\prime}\right\|$ small enough.

If $P \in X \times X$ is a point outside the diagonal, then there exists an open subset $U$ containing $P$ such that $\left.p_{t}\right|_{U}$ is a bounded form tending to zero, for $t \rightarrow 0$.

\section{The Green operator for Dirac currents}

3.1 We want a heat flux for currents as well. Let $T$ be an arbitrary current. We define the families $\left\{T_{t}\right\}_{t>0}$ of currents by

$$
T_{t}(\alpha)=T\left(P_{t}(\alpha)\right)
$$

where $P_{t}$ is the heat flux operator of theorem 2.2. The properties of the heat flux for smooth forms imply $T=\lim _{t \rightarrow 0} T_{t}$. If $[\eta]$ is the current associated to a $k$-smooth form $\eta$, then (using the notations of 2.1) we compute that

$$
\begin{aligned}
{[\eta]_{t}(\alpha) } & =\int_{X} \eta \wedge P_{t}(\alpha) \\
& =\int_{X} \eta \wedge \operatorname{pr}_{1 *}\left(p_{t} \wedge \operatorname{pr}_{2}^{*} \alpha\right) \\
& =\int_{X \times X} \operatorname{pr}_{1}^{*} \eta \wedge p_{t} \wedge \operatorname{pr}_{2}^{*} \alpha \\
& =(-1)^{k} \int_{X \times X} \operatorname{pr}_{2}^{*} \alpha \wedge p_{t} \wedge \operatorname{pr}_{1}^{*} \eta
\end{aligned}
$$

Taking into account the $\tau$-invariance of $p_{t}$ we conclude by integrating along the fibres of $\mathrm{pr}_{2}$

$$
[\eta]_{t}(\alpha)=(-1)^{k} \int_{X} \alpha \wedge P_{t}(\eta)=\int_{X} P_{t}(\eta) \wedge \alpha=\left[P_{t}(\eta)\right](\alpha)
$$

Thus, the heat flux of currents generalizes the heat flux of smooth forms.

3.2 We consider the Dirac current $\delta_{Y}$ associated to a closed submanifold $Y \subset X$. We give a formula for the heat flux of this current. In particular, we see that $\delta_{Y, t}$ is the current associated to a smooth form $\mu_{Y, t}$, for any $t>0$. By $\iota: \tilde{Y} \rightarrow X$ we denote a desingularization of $Y$. On the one hand we consider

$$
\delta_{Y, t}(\alpha)=\int_{\tilde{Y}} \iota^{*}\left(P_{t}(\alpha)\right)=\int_{\tilde{Y} \times X}\left(\iota \times \operatorname{pr}_{2}\right)^{*} p_{t} \wedge \operatorname{pr}_{2}^{*} \alpha .
$$


On the the other hand we consider the morphisms

$$
X \stackrel{\mathrm{pr}_{1}}{\longleftarrow} X \times \tilde{Y} \stackrel{\mathrm{id} \times \iota}{\longrightarrow} X \times X
$$

and the smooth form $\mu_{Y, t}$ defined by $\mu_{Y, t}:=\operatorname{pr}_{1 *}(\mathrm{id} \times \iota)^{*} p_{t}$. We have for the current associated to $\mu_{Y, t}$ the equation

$$
\left[\mu_{Y, t}\right](\alpha)=\int_{X} \operatorname{pr}_{1 *}(\mathrm{id} \times \iota)^{*} p_{t} \wedge \alpha=\int_{X \times \tilde{Y}}(\mathrm{id} \times \iota)^{*} p_{t} \wedge \operatorname{pr}_{1}^{*} \alpha
$$

Hence, the symmetry of $p_{t}$ implies that $\delta_{Y, t}$ is the current associated with the smooth form $\mu_{Y, t}$, for all $t>0$.

3.3 We want to study the smooth forms $\mu_{Y, t}$ at a point $y \in Y$, for small values of $t$. We assume that $y$ is a smooth point of $Y$, and that $Y$ is locally given by the equations $\left(z_{j}=0\right)_{j=1, \ldots, k}$. Furthermore, we assume the $z_{j}$ to be unitary coordinates at the point $y$. Since we want to express $\mu_{Y, t}$ up to a bounded form, we assume that $X=\mathbb{C}^{n}$ and $Y$ the linear subspace defined by $\left(z_{j}=0\right)_{j=1, \ldots, k}$. We take coordinates $\left\{z_{j}\right\}_{j=1, \ldots, n}$ on $X$, and $\left\{z_{j}^{\prime}\right\}_{j=k+1, \ldots, n}$ on $Y$.

Recalling our formula of 2.3 we compute that

$$
\begin{aligned}
\mu_{Y, t}(z) & =\left(\int_{Y} \prod_{j=1}^{k} \frac{\exp \left(-\frac{\left|z_{j}\right|^{2}}{4 t}\right)}{4 \pi t} \prod_{j=k+1}^{n} \frac{\exp \left(-\frac{\left|z_{j}-z_{j}^{\prime}\right|^{2}}{4 t}\right)}{4 \pi t} d x_{k+1}^{\prime} d y_{k+1}^{\prime} \ldots d x_{n}^{\prime} d y_{n}^{\prime}\right) d x_{1} d y_{1} \ldots d x_{k} d y_{k} \\
& =\prod_{j=1}^{k} \frac{\exp \left(-\frac{\left|z_{j}\right|^{2}}{4 t}\right)}{4 \pi t} d x_{1} d y_{1} \ldots d x_{k} d y_{k}
\end{aligned}
$$

Resuming our computation we obtain in a neighbourhood of a point $y \in Y$ the following local expression for the smooth form $\mu_{Y, t}$

$$
\mu_{Y, t}=\frac{\exp \left(-\frac{\sum_{j=1}^{k}\left|z_{j}\right|^{2}}{4 t}\right)}{(4 \pi t)^{k}} d x_{1} d y_{1} \ldots d x_{k} d y_{k}+\psi
$$

where $\psi$ is a $2 k$-form with bounded coefficients.

3.4 We want to extend the Green operator to Dirac currents. Let $Y \subset X$ be a smooth submanifold. Since the heat flux of currents generalizes the heat flux of smooth forms and $\mu_{Y, t}$ is smooth, the limit $\mu_{Y, \infty}:=\lim _{t \rightarrow \infty} \mu_{Y, t}$ exists and is a harmonic form. Moreover, we have for any $t>0$ the equality

$$
\mu_{Y, t}-\mu_{Y, \infty}=\Delta \int_{t}^{\infty}\left(\mu_{Y, s}-\mu_{Y, \infty}\right) d s .
$$

We want to take the limit of this equation for $t \rightarrow 0$. The limit of the left hand side exists and equals $\delta_{Y}-\mu_{Y, \infty}$. We have to investigate the existence of the integral $G(Y):=\int_{0}^{\infty}\left(\mu_{Y, s}-\mu_{Y, \infty}\right) d s$. We want to show that this integral is a form with $L^{1}$-coefficients which are well defined on the dense open subset $X \backslash Y$. To do so, we first split this integral into three summands:

$$
G(Y)=\int_{0}^{\infty}\left(\mu_{Y, s}-\mu_{Y, \infty}\right) d s=\int_{0}^{1} \mu_{Y, s} d s-\int_{0}^{1} \mu_{Y, \infty} d s+\int_{1}^{\infty}\left(\mu_{Y, s}-\mu_{Y, \infty}\right) d s .
$$

The second and the third integral on the right hand side are smooth forms. Hence we only have to show that $\int_{0}^{1} \mu_{Y, s} d s$ gives a form with $L^{1}$ coefficients. According to 2.3 it is therefore enough to show that this holds for a linear subspace $\mathbb{C}^{n-k}$ in $\mathbb{C}^{n}$. This is the object of the following lemma.

3.5 Lemma. The $\mathbb{C}^{n}$ function $f_{k, n}(z):=\int_{0}^{1} \prod_{j=1}^{k} \frac{\exp \left(\frac{-\left|z_{j}\right|^{2}}{4 t}\right)}{(4 \pi t)^{k}} d t$ is locally integrable, for any integer $k$ with $0 \leq k \leq n$. 
Proof: We first show that the $\mathbb{C}$-function $f_{1,1}$ is locally integrable. We remark that it is enough to show that the integral $\int_{|z|<1} f_{1,1} d x \wedge d y$ is a well defined finite number. By definition we have

$$
\int_{|z|<1} f_{1,1} d x \wedge d y=\int_{|z|<1} \int_{0}^{1} \frac{\exp \left(\frac{-|z|^{2}}{4 t}\right)}{4 \pi t} d t \wedge d x \wedge d y .
$$

Passing to polar coordinates around $0 \in \mathbb{C}$, we obtain

$$
\int_{|z|<1} f_{1,1} d x \wedge d y=\int_{0}^{1} \int_{0}^{1} \frac{\exp \left(\frac{-r^{2}}{4 t}\right)}{2 t} r d r d t .
$$

Using the transform $s=\frac{r^{2}}{4 t}$ this yields

$$
\int_{|z|<1} f_{1,1} d x \wedge d y=\int_{0}^{1} \int_{0}^{\frac{1}{4 t}} \exp (-s) d s d t=\int_{0}^{1}\left(1-\exp \left(-\frac{1}{4 t}\right)\right) d t<1 .
$$

Thus $f_{1,1}$ is an $L^{1}$ function.

The general case can be reduced to the special as we will see in a moment. To show that $f_{k, n}$ is $L^{1}$ it suffices to show that the integral of $f_{k, n} d x_{1} d y_{1} \ldots d x_{n} d y_{n}$ is well defined and finite on the polydisc $\left(B_{1}\right)^{n} \subset \mathbb{C}^{n}$ consisting of all $z$ with $\left|z_{j}\right|<1$, for all $j=1 \ldots n$. Analogously to the proof for $f_{1,1}$ we obtain:

$$
\begin{aligned}
\int_{\left(B_{1}\right)^{n}} f_{k, n} d x_{1} d y_{1} \ldots d x_{n} d y_{n} & =\int_{0}^{1} \int_{\left(B_{1}\right)^{n}} \prod_{j=1}^{k} \frac{\exp \left(\frac{-\left|z_{j}\right|^{2}}{4 t}\right)}{4 \pi t} d x_{1} d y_{1} \ldots d x_{n} d y_{n} d t \\
& =(2 \pi)^{n-k} \int_{0}^{1}\left(1-\exp \left(-\frac{1}{4 t}\right)\right)^{k} d t \\
& <(2 \pi)^{n-k}
\end{aligned}
$$

3.6 We have shown that for a smooth cycle $Y \subset X$ there exists a form $G(Y)$ with $L^{1}$ coefficients satisfying

$$
\delta_{Y}-\left[\mu_{Y, \infty}\right]=\Delta G(Y) .
$$

We next extend this to an arbitrary irreducible cycle $Y \subset X$. To do so we consider a resolution of singularities $\tilde{Y} \rightarrow Y$ and the following situation

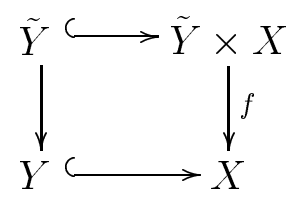

We choose a Kähler metric on $\tilde{Y}$ and equip $\tilde{Y} \times X$ with the product metric. For the smooth $\tilde{Y} \times X$-cycle $\tilde{Y}$ we have a form $G(\tilde{Y})$ with $L^{1}$-coefficients such that the equality $\delta_{\tilde{Y}}-\left[\mu_{\tilde{Y}, \infty}\right]=$ $\Delta G(\tilde{Y})$ of currents holds. We apply the push forward $f_{*}$ of currents to this equality. By definition $f_{*} \delta_{\tilde{Y}}=\delta_{Y}$. The harmonic form $\mu_{\tilde{Y}, \infty}$ pushes forward to a harmonic form because we took the product metric on $\tilde{Y} \times X$. By the same reason $f_{*} \Delta G(\tilde{Y})=\Delta f_{*} G(\tilde{Y})$. Thus setting $G(Y):=f_{*} G(\tilde{Y})$ we obtain the desired equality

$$
\delta_{Y}-\left[\mu_{Y, \infty}\right]=\Delta G(Y),
$$

for an arbitrary irreducible cycle $Y \subset X$.

3.7 We show here that for a divisor $Y \subset X$ the current $\int_{0}^{1} \mu_{Y, t} d t$ is of logarithmic growth along $Y$, i.e., we show that the function

$$
f(a):=\int_{0}^{1} \frac{\exp \left(-\frac{a}{4 t}\right)}{t} d t
$$


for $a \in[0,1]$ behaves like $-\ln (a)$ up to something bounded. We see that $\lim _{a \rightarrow 0} f(a)=\infty$. Using the new coordinate $s=\frac{a}{4 t}$ the defining equation for $f$ yields

$$
f(a)=\int_{\frac{a}{4}}^{\infty} \frac{\exp (-s)}{s} d s
$$

The number $C_{1}:=\int_{1}^{\infty} \frac{\exp (-s)}{s} d s$ is obviously finite $\left(C_{1} \approx 0.22\right)$. The function $g(s)=\frac{\exp (-s)-1}{s}$ is holomorphic on $\mathbb{C}$. Thus, $g(s)$ is a bounded function at the unit interval $[0,1]$. Consequently, we obtain

$$
\begin{aligned}
f(a)-C_{1} & =\int_{\frac{a}{4}}^{1} \frac{d s}{s}+\int_{\frac{a}{4}}^{1} g(s) d s \\
& =\ln (4)-\ln (a)+\int_{\frac{a}{4}}^{1} g(s) d s .
\end{aligned}
$$

Resuming our computations we obtain: For $a \in[0,1]$ we have

$$
\int_{0}^{1} \frac{\exp \left(-\frac{a}{4 t}\right)}{t} d t=-\ln (a)+O(1)
$$

3.8 Here we compute the growth for the Green operator for a cycle $Y \subset X$ of codimension $k>1$. Thus, we have to investigate the function

$$
f_{k}(a):=\int_{0}^{1} \frac{\exp \left(\frac{-a}{4 t}\right)}{t^{k}} d t
$$

Again we use the coordinate $s=\frac{a}{4 t}$ and obtain

$$
\begin{aligned}
f_{k}(a) & =\left(\frac{4}{a}\right)^{k-1} \cdot \int_{\frac{a}{4}}^{\infty} \exp (-s) s^{k-2} d s \\
& =\left(\frac{4}{a}\right)^{k-1} \cdot\left(\int_{0}^{\infty} \exp (-s) s^{k-2} d s-\int_{0}^{\frac{a}{4}} \exp (-s) s^{k-2} d s\right) \\
& =\left(\frac{4}{a}\right)^{k-1} \cdot\left((k-2) !-O\left(a^{k-1}\right)\right) \\
& =(k-2) !\left(\frac{4}{a}\right)^{k-1}+O(1) .
\end{aligned}
$$

We summarize these computations in the next result.

3.9 Lemma. If $Y \subset X$ is locally defined by the equation $z=0$, then the Green operator $G(Y)$ of the divisor $Y$ is given by

$$
G(Y)=\frac{\log \left(|z|^{2}\right)}{8 \pi i} d z \wedge d \bar{z}+\text { some smooth form. }
$$

If the cycle $Y \subset X$ is locally defined by the equations $\left(z_{1}=z_{2}=\ldots z_{k}=0\right)$, then the Green operator $G(Y)$ has the local form

$$
\left(\frac{i}{2 \pi}\right)^{k} \cdot \frac{(k-2) !}{4}\left(\left|z_{1}\right|^{2}+\ldots+\left|z_{k}\right|^{2}\right)^{1-k} d z_{1} \wedge d \bar{z}_{1} \wedge d z_{2} \wedge \ldots \wedge d \bar{z}_{k}+\text { some smooth form }
$$




\section{The canonical harmonic Green current}

4.1 Lemma. Let $Y$ be a closed subvariety of a smooth Kähler manifold $X$ and $G(Y)$ its Green operator. Then $G(Y)$ is closed with respect to $d$, $\partial$, and $\bar{\partial}$.

Proof: We start with the equation $\delta_{Y}-\left[\mu_{Y, \infty}\right]=\Delta G(Y)$ of 3.6. Since the left hand side of this equation is $d$-closed, we obtain $d \Delta G(Y)=0$. Since $\Delta$ and $d$ commute, we derive that $\Delta(d G(Y))=0$. Thus, $d G(Y)$ is an exact current and harmonic. This implies $d G(Y)=0$, because of the Hodge decomposition.

4.2 Definition. We set $d^{c}:=\frac{1}{4 \pi i}(\partial-\bar{\partial})$.

A current $g_{Y}$ is a Green current for the cycle $Y \subset X$ if $\delta_{Y}+d d^{c} g_{Y}$ is the current associated with a smooth form.

The current $g_{Y}$ is a harmonic Green current if $\delta_{Y}+d d^{c} g_{Y}$ is the current associated with a harmonic form.

4.3 Theorem. Let $Y$ be an irreducible subvariety of the compact Kähler manifold $Y$. Then $4 \pi \Lambda G(Y)$ is a harmonic Green current.

Proof: Again we take the equation $\delta_{Y}-\left[\mu_{Y, \infty}\right]=\Delta G(Y)$ of 3.6 as our starting point. We remark that $\mu_{Y, \infty}$ is the harmonic projection of $\delta_{Y}$. This yields

$$
\begin{array}{rlrl}
{\left[\mu_{Y, \infty}\right]-\delta_{Y}} & =-\Delta G(Y) & \\
& =-2\left(\bar{\partial} \bar{\partial}^{*}+\bar{\partial}^{*} \bar{\partial}\right) G(Y) & & \\
& =-2 \bar{\partial} \bar{\partial}^{*} G(Y) & & (G(Y) \text { is } \bar{\partial} \text { closed) } \\
& =-2 i \bar{\partial}(\partial \Lambda-\Lambda \partial) G(Y) & & \text { (Hodge identity) } \\
& =-2 i \bar{\partial} \partial \Lambda G(Y) & & (G(Y) \text { is } \partial \text { closed) } \\
& =\frac{1}{2 \pi i} \bar{\partial} \partial(4 \pi \Lambda G(Y)) & & \\
& =d^{c}(4 \pi \Lambda G(Y)) &
\end{array}
$$

Thus, $4 \pi \Lambda G(Y)$ is a harmonic Green current for the cycle $Y$.

4.4 Lemma. The harmonic projection of $4 \pi \Lambda G(Y)$ is zero.

Proof: We have $\Lambda G(Y)=\lim _{t \rightarrow 0} \Lambda G\left(\mu_{Y, t}-\mu_{Y, \infty}\right)$. Hence, it suffices to show that the harmonic projection of $\Lambda G\left(\mu_{Y, t}-\mu_{Y, \infty}\right)$ is zero for all $t>0$. This is equivalent to showing that $\Lambda G\left(\mu_{Y, t}-\right.$ $\left.\mu_{Y, \infty}\right)$ is in the orthogonal complement of the harmonic forms. We set $\alpha:=G\left(\mu_{Y, t}-\mu_{Y, \infty}\right)$. We have to show that the $L^{2}$-scalar product $\left.(\Lambda \alpha, \beta)=\int_{X}<\Lambda \alpha, \beta\right\rangle \omega^{n}$ vanishes for all harmonic forms $\beta$. Now $(\Lambda \alpha, \beta)=(\alpha, L \beta)$. Thus, it is enough to show that $L \beta$ is harmonic because $\alpha$ has harmonic projection equal to zero. However, the Hodge identities imply $L \Delta=\Delta L$. Hence, $\Delta(L \beta)=0$ which implies that $L \beta$ is a harmonic form.

Since it depends only on $X, \omega$ and $Y$ we call $4 \pi \Lambda G(Y)$ the canonical harmonic Green current. Using the Hodge decomposition for currents we are able to describe all Green currents for the cycle $Y$.

4.5 Lemma (see [2] 1.2.2 (iii)). Let $g_{Y}$ and $g_{Y}^{\prime}$ be two harmonic Green currents for the cycle $Y \subset X$ of codimension $k$. Then their difference $g_{Y}-g_{Y}^{\prime}$ is contained in the space $\mathcal{H}^{k-1, k-1}(X)+$ $\partial D^{k-2, k-1}(X)+\bar{\partial} D^{k-1, k-2}(X)$.

Proof: We set $g_{0}=g_{Y}-g_{Y}^{\prime}$. By definition we have $\partial \bar{\partial} g_{0}=0$. Using the Hodge decomposition for currents, we express $\bar{\partial} g_{0}$ as

$$
\bar{\partial} g_{0}=h_{1}+\partial g_{1}+\partial^{*} \tilde{g}_{1}
$$

where $h_{1} \in \mathcal{H}^{k-1, k}(X), g_{1} \in D^{k-2, k}(X)$, and $\tilde{g}_{1} \in D^{k, k}(X)$. Since $\partial \bar{\partial} g_{0}=0$, we have $\partial^{*} \tilde{g}_{1}$ is $\partial$-closed. Obviously, $\partial^{*} \tilde{g}_{1}$ is $\partial^{*}$-closed. Thus, $\partial^{*} \tilde{g}_{1}$ is harmonic. Hence, we have $\partial^{*} \tilde{g}_{1}=0$. The 
now resulting equality $\bar{\partial} g_{0}=h_{1}+\partial g_{1}$ yields $\partial \bar{\partial} g_{1}=0$. Thus, we can repeat the argument to obtain a sequence $\left(g_{l}\right)_{l \geq 0}$ of currents satisfying

$$
\bar{\partial} g_{l-1}=h_{l}+\partial g_{l} \quad \text { with } g_{l} \in D^{k-1-l, k-1+l}(X) \text { and } h_{l} \in \mathcal{H}^{k-l, k-1+l}(X) .
$$

We end up with $\bar{\partial} g_{k-1}=h_{k}$. The Hodge decomposition implies that a $\bar{\partial}$-exact current which is harmonic has to be zero. So we conclude $\bar{\partial} g_{k-1}=0$.

By applying the Hodge decomposition to $g_{k-1}$, we obtain

$$
g_{k-1}=H_{k-1}+\bar{\partial} G_{k-1}+\bar{\partial}^{*} \tilde{G}_{k-1} .
$$

As before, from $\bar{\partial} g_{k-1}=0$ we conclude $\bar{\partial}^{*} \tilde{G}_{k-1}=0$. Now we compute $\bar{\partial} g_{k-2}$ using the above formula:

$$
\bar{\partial} g_{k-2}=h_{k-1}+\partial\left(H_{k-1}+\bar{\partial} G_{k-1}\right)=h_{k-1}+\partial \bar{\partial} G_{k-1} \cdot
$$

This yields $\bar{\partial}\left(g_{k-2}+\partial G_{k-1}\right)=h_{k-1}$.

Repeating this process we obtain $h_{k-1}=0, g_{k-2}+\partial G_{k-1}=H_{k-2}+\bar{\partial} G_{k-2}$, and $\bar{\partial} g_{k-3}=$ $h_{k-2}+\partial \bar{\partial} G_{k-2}$. Thus, we end up with $g_{0}=H_{0}-\partial G_{1}+\bar{\partial} G_{0}$, which proves the lemma.

4.6 Corollary. Let $g_{Y}$ and $g_{Y}^{\prime}$ be two Green currents for the cycle $Y \subset X$ of codimension $k$. Then the difference $g_{Y}-g_{Y}^{\prime}$ is contained in the space $A^{k-1, k-1}(X)+\partial D^{k-2, k-1}(X)+$ $\bar{\partial} D^{k-1, k-2}(X)$.

Proof: Let $\delta_{Y}+d d^{c} g_{Y}=\mu_{Y}$, and $\delta_{Y}+d d^{c} g_{Y}^{\prime}=\mu_{Y}^{\prime}$. Then $\mu_{Y}-\mu_{Y}^{\prime}$ is a smooth form representing the trivial class in cohomology. Thus, repeating the argument of theorem 4.3 we see that $\mu_{Y}-\mu_{Y}^{\prime}$ is of the form $d d^{c} \alpha$, for some smooth $(k-1, k-1)$-form $\alpha$.

4.7 From the local computations of lemma 3.9 we obtain that the canonical Green current $g_{Y}$ of a smooth divisor $Y \subset X$ locally given by $z=0$ is of the form

$$
g_{Y}=-\log \left(\|z\|^{2}\right)+\text { some smooth function. }
$$

If $Y$ is a smooth cycle given by $\left(z_{1}=\ldots=z_{k}=0\right)$, then lemma 3.9 yields that the canonical harmonic Green current $g_{Y}$ equals

$$
(k-2) ! \cdot\left(\frac{i}{2 \pi\|z\|^{2}}\right)^{k-1} \sum_{j=1}^{k} d z_{1} \wedge d \bar{z}_{1} \wedge \ldots \wedge d \bar{z}_{j-1} \wedge d z_{j+1} \wedge \ldots \wedge d \bar{z}_{k}
$$

plus some smooth $(k-1, k-1)$-form.

\section{The wedge product of Dirac currents}

5.1 Let $Y$ and $Z$ be two analytic cycles in $X$. We want to define the wedge product $\delta_{Y} \wedge \delta_{Z}$ and relate it to the Dirac current of their intersection product. However, we can only wedge a current with a smooth form. Hence, we can wedge a current only with those currents which fulfill certain conditions. The idea is that in order to intersect $Y$ and $Z$ we intersect $Y \times Z$ with the diagonal $\Delta_{X}$ in $X \times X$. This diagonal is the limit of a family of smooth currents: the heat kernel.

Let $\tilde{Y} \rightarrow Y$ and $\tilde{Z} \rightarrow Z$ be two desingularizations of our cycles. We consider the following situation

$$
\tilde{Y} \times \tilde{Z} \stackrel{f}{\longrightarrow} X \times X \stackrel{\mathrm{pr}_{1}}{\longrightarrow} X .
$$

For $t>0$ we define $\left(\delta_{Y} \wedge \delta_{Z}\right)_{t}$ by

$$
\left(\delta_{Y} \wedge \delta_{Z}\right)_{t}(\alpha):=\int_{\tilde{Y} \times \tilde{Z}} f^{*}\left(p_{t} \wedge \operatorname{pr}_{1}^{*} \alpha\right)
$$


where $p_{t}$ is the heat kernel on $X \times X$. We say that $\delta_{Y}$ and $\delta_{Z}$ are wedgeable if for all smooth forms $\alpha$ on $X$ the limit

$$
\left(\delta_{Y} \wedge \delta_{Z}\right)(\alpha)=\lim _{t \rightarrow 0}\left(\delta_{Y} \wedge \delta_{Z}\right)_{t}(\alpha)
$$

exists.

5.2 It might be useful to see that for any smooth form $\alpha$ the equality $\left(\delta_{Y} \wedge \delta_{Z}\right)_{t}(\alpha)=\delta_{Y}\left(\mu_{Z, t} \wedge \alpha\right)$ holds. Hence, we have $\left(\delta_{Y} \wedge \delta_{Z}\right)_{t}=\delta_{Y} \wedge \mu_{Z, t}$. In order to obtain this equality of currents, we consider the following commutative diagram.

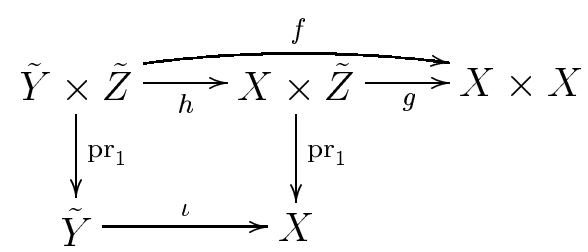

Recalling the definitions of $\mu_{Z, t}$ and $\left(\delta_{Y} \wedge \delta_{Z}\right)_{t}$, we obtain:

$$
\begin{aligned}
\delta_{Y}\left(\mu_{Z, t} \wedge \alpha\right) & =\int_{\tilde{Y}} \iota^{*}\left(\operatorname{pr}_{1 *}\left(g^{*} p_{t}\right)\right) \wedge \iota^{*} \alpha=\int_{\tilde{Y}} \operatorname{pr}_{1 *} h^{*} g^{*} p_{t} \wedge \iota^{*} \alpha \\
& =\int_{\tilde{Y} \times \tilde{Z}} f^{*} p_{t} \wedge \operatorname{pr}_{1}^{*} \iota^{*} \alpha=\left(\delta_{Y} \wedge \delta_{Z}\right)_{t}(\alpha) .
\end{aligned}
$$

Thus, we have shown the asserted equality of currents.

We say that $Y$ and $Z$ intersect properly if their intersection has the expected dimension. We will need the following result for the computation of intersection multiplicities.

5.3 Lemma. Let $\left\{f_{i}\right\}_{i=1, \ldots, n}$ be a regular sequence of holomorphic functions in a neighbourhood of $0 \in \mathbb{C}^{n}$, and $g$ be a smooth function on $\mathbb{C}^{n}$. We define $F(t)$ by

$$
F(t)=\frac{i^{n}}{(8 \pi t)^{n}} \int_{\left(B_{\varepsilon}\right)^{n}} g(z) \exp \left(\frac{-\left(\left|f_{1}\right|^{2}+\ldots+\left|f_{n}\right|^{2}\right)}{4 t}\right) d f_{1} \wedge d \bar{f}_{1} \wedge \ldots \wedge d f_{n} \wedge d \bar{f}_{n} .
$$

(1) Then for $\varepsilon>0$ small enough we have $\lim _{t \rightarrow 0} F(t)=g(0) \cdot \operatorname{mult}_{0}\left(f_{1}, \ldots, f_{n}\right)$.

(2) If the multiplicity of 0 is one, then there exists constants a and $b$ not depending on $g$, $\varepsilon$ and $t$ such that

$$
|F(t)-F(0)| \leq \frac{a t}{\varepsilon^{2}}\|g\|_{\max }+b \sqrt{t}|| d g \|_{\max } .
$$

Proof: (1) We first compute the above integral in case $g \equiv 1$, and $f_{i}=z_{i}$, for all $i=1, \ldots, n$. To this end, we compute using polar coordinates

$$
\lim _{t \rightarrow 0} \int_{B_{\varepsilon}} \frac{i \exp \left(\frac{-|z|^{2}}{4 t}\right)}{8 \pi t} d z d \bar{z}=\lim _{t \rightarrow 0} \int_{0}^{2 \pi} \int_{0}^{\varepsilon} \frac{\exp \left(\frac{-r^{2}}{4 t}\right)}{4 \pi t} r d r d \phi=\lim _{t \rightarrow 0} \int_{0}^{\varepsilon} \exp \left(\frac{-r^{2}}{4 t}\right) \frac{r d r}{2 t}
$$

Applying the transformation $s=\frac{r^{2}}{4 t}$ we find that

$$
\lim _{t \rightarrow 0} \int_{B_{\varepsilon}} \frac{i \exp \left(\frac{-|z|^{2}}{4 t}\right)}{8 \pi t} d z d \bar{z}=\lim _{t \rightarrow 0} \int_{0}^{\frac{\varepsilon^{2}}{4 t}} \exp (-s) d s=\lim _{t \rightarrow 0}\left(1-\exp \left(\frac{-\varepsilon^{2}}{4 t}\right)\right)=1 .
$$

Using this formula $n$-times, we find that the assertion is true for any $\varepsilon$ if $f_{i}=z_{i}$, and $g \equiv 1$. Since $g$ was assumed to be continuous, we find that the asserted formula holds for a continuous function $g$ if $f_{i}=z_{i}$, for $i=1, \ldots, n$. 
After these technical preparations the proof of the first part is easy. We take (if necessary after passing to a smaller $\varepsilon) r_{1}<r_{2}$ such that $f\left(\left(B_{\varepsilon}\right)^{n}\right) \subset\left(B_{r_{2}}\right)^{n}$, and $f^{-1}\left(B_{r_{1}}\right)^{n} \subset\left(B_{\varepsilon}\right)^{n}$ holds. We remark that it is enough to compute the integral on the dense open subset where $f=\left(f_{1}, \ldots, f_{n}\right)$ is unramified. On that subset it gives a mult ${ }_{0}\left(f_{1}, \ldots, f_{n}\right): 1$ cover over the base. Thus, by passing to the coordinates $z_{i}$ on $f\left(B_{\varepsilon}\right)$ we obtain the required equality.

(2) We show this only for $f_{j}=z_{j}$, because the general case is as before easily deduced from this case. We need to find an upper bound for $\frac{\partial F(t)}{\partial t}$. We set $\|z\|^{2}=\left|z_{1}\right|^{2}+\ldots+\left|z_{n}\right|^{2}$. By $d \mu$ we denote the Lebesgue measure on $\left(B_{\varepsilon}\right)^{n}$ while $d \nu$ is the Lebesgue measure on $S^{2 n-1}$.

$$
\begin{aligned}
\frac{\partial F(t)}{\partial t} & =\frac{1}{(4 \pi)^{n}} \int_{\left(B_{\varepsilon}\right)^{n}} g(z) \exp \left(\frac{-|z|^{2}}{4 t}\right)\left(\frac{|z|^{2}}{4 t^{n+2}}-\frac{n}{t^{n+1}}\right) d \mu \\
& =\frac{1}{(4 \pi)^{n}} \int_{S^{2 n-1}} \int_{0}^{\varepsilon} g(r, \phi) \exp \left(\frac{-r^{2}}{4 t}\right)\left(\frac{r^{2 n+1}}{4 t^{n+2}}-\frac{n r^{2 n-1}}{t^{n+1}}\right) d r d \nu
\end{aligned}
$$

Next we try to find a bound for $M(t):=\int_{0}^{\varepsilon} g(r, \phi) \exp \left(\frac{-r^{2}}{4 t}\right)\left(\frac{r^{2 n+1}}{4 t^{n+2}}-\frac{n r^{2 n-1}}{t^{n+1}}\right) d r$.

We will use the function $H(r, t):=\frac{-r^{2 n}}{2 t^{n+1}} \exp \left(\frac{-r^{2}}{4 t}\right)$ which satisfies

$$
\frac{\partial}{\partial r} H(r, t)=\exp \left(\frac{-r^{2}}{4 t}\right)\left(\frac{r^{2 n+1}}{4 t^{n+2}}-\frac{n r^{2 n-1}}{t^{n+1}}\right) \text {. }
$$

For fixed $r$ we see that the function $t \mapsto H(r, t)$ is a non-positive smooth function, for $t \in[0, \infty)$ with minimum $\frac{-\exp (-n-1)(4 n+4)^{n+1}}{2 r^{2}}$. Using the transformation $s=\frac{r^{2}}{4 t}$ we obtain

$$
\begin{aligned}
\int_{0}^{\varepsilon}|H(r, t)| d r & =\frac{4^{n}}{2 \sqrt{t}} \int_{0}^{\frac{\varepsilon^{2}}{4 t}} s^{n-\frac{1}{2}} \exp (-s) d s \\
& <\frac{4^{n}}{2 \sqrt{t}} \int_{0}^{\infty} s^{n-\frac{1}{2}} \exp (-s) d s \\
& <\frac{4^{n}}{2 \sqrt{t}} \Gamma\left(n+\frac{1}{2}\right) .
\end{aligned}
$$

Having this in mind, we deduce the following inequality:

$$
\begin{aligned}
M(t) & =\int_{0}^{\varepsilon} g(r, \phi) \frac{\partial}{\partial r} H(r, t) d r \\
& =H(\varepsilon, t) g(\varepsilon, \phi)-H(0, t) g(0, \phi)-\int_{0}^{\varepsilon} H(r, t) \frac{\partial}{\partial r} g(r, \phi) d r \\
& \leq|H(\varepsilon, t)| \cdot\|g\|_{\max }+\int_{0}^{\varepsilon}|H(r, t)| d r \cdot\|d g\|_{\max } \\
& \leq \frac{\exp (-n-1)(4 n+4)^{n+1}}{2 \varepsilon^{2}}\|g\|_{\max }+\frac{4^{n}}{2 \sqrt{t}} \Gamma\left(n+\frac{1}{2}\right)\|d g\|_{\max } .
\end{aligned}
$$

From this we directly conclude the existence of constants $a$ and $b$ such that

$$
\left|\frac{\partial F(t)}{\partial t}\right| \leq \frac{a}{\varepsilon^{2}}\|g\|_{\max }+\frac{b}{2 \sqrt{t}}\|d g\|_{\max } .
$$

This inequality yields the assertion (2).

5.4 Lemma (see [2] 2.1.5). If $Y$ and $Z$ intersect properly, then the Dirac currents $\delta_{Y}$ and $\delta_{Z}$ are wedgeable. Their wedge product $\delta_{Y} \wedge \delta_{Z}$ equals the Dirac current $\delta_{Y \cap Z}$ of their intersection product $Y \cap Z$ (including multiplicities).

Proof: If the sum of the dimensions of $Y$ and $Z$ is less than the dimension of $X$, then $\left(\delta_{Y} \wedge \delta_{Z}\right)_{t} \equiv 0$, for all $t>0$. Thus, in this case there is nothing to prove. 
Now we treat the case when $\operatorname{dim}(Y)+\operatorname{dim}(Z)=\operatorname{dim}(X)$. Take a point $P=(0,0) \in Y \times Z$. We assume that $P$ is a smooth point of $Y \times Z$. Let $f_{1}, \ldots, f_{n}$ be the pull back of functions defining the diagonal in $X \times X$ in a neighbourhood of $P$. Then the pull back of the heat kernel to $\tilde{Y} \times \tilde{Z}$ is given by (see 2.3 )

$$
f^{*} p_{t}=\frac{i^{n}}{(8 \pi t)^{n}} \exp \left(\frac{-\left(\left|f_{1}\right|^{2}+\ldots+\left|f_{n}\right|^{2}\right)}{4 t}\right) d f_{1} \wedge d \bar{f}_{1} \wedge \ldots \wedge d f_{n} \wedge d \bar{f}_{n}+\rho
$$

where $\rho$ denotes a $(n, n)$-form with bounded coefficients which tend to zero for $t \rightarrow 0$ outside of $P$. Thus, by part (1) of lemma 5.3, we have $\delta_{Y} \wedge \delta_{Z}=\delta_{Y \cap Z}$.

If $P$ is not a smooth point of the $Y \times Z$ we proceed as follows. We consider a family of small disturbances of the diagonal. Precisely we consider the family $\left(P_{s}\right)_{s \in[0,1]}$ of zero cycles in $Y \times Z$ where $P_{s}$ is the zero locus of $\left(f_{1}+s=0, f_{2}=0, \ldots, f_{n}=0\right)$. Without loss of generality we can assume that for $s \in(0,1] P_{s}$ is a smooth 0-cycle contained in the smooth locus of $Y \times Z$ and in a $\varepsilon$-ball around $P$. Thus, $P_{s}=P_{s, 1} \cup P_{s, 2} \cup \ldots P_{s, m}$ where $m=\operatorname{mult}_{P}(Y, Z)$. Let $B$ be an $\varepsilon$-ball around $P$. We consider the function

$$
F(t, s):=\int_{B} f^{*}\left(\frac{i^{n}}{(8 \pi t)^{n}} \exp \left(\frac{-\left(\left|f_{1}+s\right|^{2}+\ldots+\left|f_{n}\right|^{2}\right)}{4 t}\right) d f_{1} \wedge d \bar{f}_{1} \wedge \ldots \wedge d f_{n} \wedge d \bar{f}_{n}+\rho\right)
$$

defined for $s \in[0,1]$ and $t>0$ with $f_{i}$ and $\rho$ as above. The smoothness of $P_{s}$ implies that

$$
\lim _{t \rightarrow 0} F(t, s)=\sum_{k=1}^{m} g\left(P_{s, k}\right)
$$

for $s>0$. Thus, we can extend $F$ to a continuous function to all points $(0, s)$ when $s>0$. Since $g$ is bounded we can bound $F(0, s)$. The second part of lemma 5.3 guarantees that $F$ can be extented to a continuous function for $t \geq 0$ and $s \in[0,1]$.

The case $\operatorname{dim}(Y)+\operatorname{dim}(Z)>\operatorname{dim}(X)$ will be reduced to the above case. Let $\left(T_{l}\right)_{l=1 \ldots k}$ be the irreducible components of $Y \cap Z$.

Since $p_{t}$ is tending to zero outside of the $T_{l}$ we can restrict our integration to tubular neighbourhoods of the smooth locus of the $T_{l}$. These neighbourhoods are locally of type $T_{l} \times\left(B_{\varepsilon}\right)^{m}$. We consider the following morphisms

$$
T_{l} \stackrel{\iota}{\longrightarrow} T_{l} \times\left(B_{\varepsilon}\right)^{m} \stackrel{f}{\longrightarrow} X \times X \stackrel{\mathrm{pr}_{1}}{\longrightarrow} X
$$

Applying the results of the above case we obtain

$$
\lim _{t \rightarrow 0} \int_{T_{l} \times\left(B_{\varepsilon}\right)^{m}} f^{*}\left(p_{t} \wedge \operatorname{pr}_{1}^{*} \alpha\right)=\operatorname{mult}_{T_{l}}(Y, Z) \int_{T_{l}} \iota^{*} f^{*} \operatorname{pr}_{1}^{*} \alpha .
$$

Thus, we obtain the asserted formula for the wedge product.

\section{$6 \quad$ The $*$-Product of Green currents}

6.1 Let $Y$ and $Z$ be two $X$-cycles which intersect properly. Furthermore, let $g_{Y}$ and $g_{Z}$ be two Green currents for $Y$ and $Z$. Hence, we have the following equalities of currents

$$
\left[\mu_{Y}\right]-\delta_{Y}=d d^{c} g_{Y} \quad \text { and } \quad\left[\mu_{Z}\right]-\delta_{Z}=d d^{c} g_{Z}
$$

The next lemma shows how a Green current for the cycle $Y \cap Z$ can be computed from these data. To do so, we have to restrict ourselves to Green currents which behave well. 
6.2 Assumption. The Green current $g_{Y}$ is locally given by forms with $L^{1}$-coefficients. These coefficients are smooth on a dense open subset whose restriction to $Z$ is dense too. Furthermore, the restriction of $g_{Y}$ to $Z$ is assumed to be a form with $L^{1}$-coefficients on $Z$.

The local formulae of 4.7 show that the canonical harmonic Green current $g_{Y}$ of $Y$ fulfills this assumption when $Y$ and $Z$ intersect properly.

6.3 Definition/Lemma (see 2.1.4 in [2]). Let $Y$ and $Z$ be two properly intersecting $X$ cycles. Moreover, let $g_{Y}$ and $g_{Z}$ be Green currents for $Y$ and $Z$ satisfying 6.2. Then the current $g_{Y} * g_{Z}:=\mu_{Y} \wedge g_{Z}+\left.g_{Y}\right|_{Z}$ is well defined and fulfills

$$
\left[\mu_{Y} \wedge \mu_{Z}\right]-\delta_{Y \cap Z}=d d^{c} g_{Y} * g_{Z} .
$$

We call $g_{Y} * g_{Z}$ the $*$-product of $g_{Y}$ and $g_{Z}$.

Proof: We take the equality $\left[\mu_{Y}\right]-\delta_{Y}=d d^{c} g_{Y}$ and wedge it with the current $\delta_{Z}$. To do so, we wedge this equality with $\mu_{Z, t}$ and consider the limit, for $t \rightarrow 0$. Since $Y$ and $Z$ intersect properly, we conclude by lemma 5.4 and remark 5.2 that $\lim _{t \rightarrow 0} \delta_{Y} \wedge \mu_{Z, t}=\delta_{Y \cap Z}$. For a smooth form $\alpha$ we have

$$
\begin{aligned}
\lim _{t \rightarrow 0}\left(\left(d d^{c} g_{Y}\right) \wedge \mu_{Z, t}\right)(\alpha) & =\lim _{t \rightarrow 0} \int_{X} \mu_{Z, t} \wedge g_{Y} \wedge d d^{c} \alpha \\
& =\lim _{t \rightarrow 0} \int_{X \times \tilde{Z}}\left(\operatorname{pr}_{1} \times \iota\right)^{*} p_{t} \wedge \operatorname{pr}_{1}^{*}\left(g_{Y} \wedge d d^{c} \alpha\right) \\
& =\left.\lim _{t \rightarrow 0} \int_{\tilde{Z}} P_{t}\left(g_{Y} \wedge d d^{c} \alpha\right)\right|_{Z} \\
& =d d^{c}\left(\left.g_{Y}\right|_{Z}\right)(\alpha)
\end{aligned}
$$

where $\iota: \tilde{Z} \rightarrow X$ denotes a desingularization of $Z$. The assumption 6.2 was needed for integrating along the fibres of $X \times \tilde{Z}$, for both projections. Thus, we obtain

$$
\mu_{Y} \wedge \delta_{Z}-\delta_{Y \cap Z}=\left.d d^{c} g_{Y}\right|_{Z}
$$

Wedging the equality $\left[\mu_{Z}\right]-\delta_{Z}=d d^{c} g_{Z}$ with $\mu_{Y}$ we obtain

$$
\left[\mu_{Y} \wedge \mu_{Z}\right]-\mu_{Y} \wedge \delta_{Z}=\mu_{Y} \wedge d d^{c} g_{Z}=d d^{c} \mu_{Y} \wedge g_{Z}
$$

Adding both equalities proves the lemma.

\section{Examples}

7.1 A point on the projective line. Let $X$ be the projective line $\mathbb{P}_{\mathbb{C}}^{1}$ with the usual open cover $X=U_{0} \cup U_{1}$ and coordinate change $z_{0}=z_{1}^{-1}$. We will see here that on a Riemannian surface it is easy to check if a given current is the canonical harmonic current for a divisor. The Kähler form $\omega$ corresponding to the Fubini-Study metric is given by

$$
\omega=d d^{c}\left(\log \left(1+z_{0} \bar{z}_{0}\right)\right)=\frac{i d z_{0} \wedge d \bar{z}_{0}}{2 \pi\left(1+z_{0} \bar{z}_{0}\right)^{2}} .
$$

Let $Y$ be the divisor defined by $z_{0}=0$. The canonical harmonic current $g_{Y}$ of $Y$ is

$$
g_{Y}=\log \left(1+\frac{1}{z_{0} \bar{z}_{0}}\right)-1
$$

To see that $g_{Y}$ is the canonical harmonic current of $Y$ we first remark that $g_{Y}$ extends to a smooth function on $U_{1}$. On the open set $U_{0}$ we have

$$
\begin{aligned}
d d^{c} g_{Y} & =d d^{c} \log \left(\frac{1+z_{0} \bar{z}_{0}}{z_{0} \bar{z}_{0}}\right) \\
& =d d^{c} \log \left(1+z_{0} \bar{z}_{0}\right)-d d^{c} \log \left(z_{0} \bar{z}_{0}\right) \\
& =\omega-\delta_{Y} .
\end{aligned}
$$


The latter equality holds because of the Poincaré-Lelong lemma. Since $\omega$ is harmonic, $g_{Y}$ differs according to lemma 4.5 only by a constant $c$ from the canonical harmonic current. Applying lemma 4.4 we find that this constant $c$ is given by $c=\int_{X} g_{Y} \omega$. To conclude that $g_{Y}$ is the canonical harmonic current for $Y$ we have to show that $\int_{X} g_{Y} \omega=0$. This is a straightforward calculation.

7.2 A point on an elliptic curve. We consider the elliptic curve $X_{\tau}:=\mathbb{C} / \Lambda_{\tau}$ where $\Lambda_{\tau}$ is the cocompact lattice $\mathbb{Z}+\tau \mathbb{Z}$ and $\tau$ is a complex number with $v:=\Im(\tau)>0$. The volume of $X$ with respect to the Kähler form $d x \wedge d y$ equals $v$. We want to consider the cycle $0 \in X$. Using Fourier analysis, we express the current $\delta_{0}$ as

$$
\delta_{0}=\frac{1}{v} \sum_{\lambda \in \Lambda_{\tau}} f_{\lambda} d x \wedge d y
$$

where $f_{\lambda}(z)=\exp \left(\frac{2 \pi i}{v} \Im(z \cdot \bar{\lambda})\right)$. These $f_{\lambda}$ are the eigenvectors of the Laplacian on $X_{\tau}$. We have

$$
\Delta\left(f_{\lambda} d x \wedge d y\right)=\frac{4 \pi^{2}}{v^{2}}|\lambda|^{2} f_{\lambda} d x \wedge d y
$$

Consequently, we can easily write down the following equations: The heat flux of $\delta_{0}$ equals

$$
\mu_{0, t}=\frac{1}{v} \sum_{\lambda \in \Lambda_{\tau}} \exp \left(-\frac{4 \pi^{2}|\lambda|^{2}}{v^{2}} t\right) f_{\lambda} d x \wedge d y
$$

and its harmonic projection is $\mu_{0, \infty}=\frac{1}{v} d x \wedge d y$. The Green operator $G(0)$ of $\delta_{0}$ is

$$
G(0)=v \sum_{\lambda \in \Lambda_{\tau} \backslash\{0\}} \frac{f_{\lambda} d x \wedge d y}{4 \pi^{2}|\lambda|^{2}} .
$$

Eventually, we obtain the canonical harmonic Green current $g_{0}$

$$
g_{0}=\frac{v}{\pi} \sum_{\lambda \in \Lambda_{\tau} \backslash\{0\}} \frac{f_{\lambda}}{|\lambda|^{2}}
$$

7.3 A point in $\mathbb{C}^{2}$. Here we take $X$ to be $\mathbb{C}^{2}$ with Kähler form $\omega=i\left(d z_{1} \wedge d \bar{z}_{1}+d z_{2} \wedge d \bar{z}_{2}\right)$, and $Y$ to be the point $0 \in \mathbb{C}^{2}$. There is no harmonic projection of the current $\delta_{Y}$, but for any $t>0$ we have the equality

$$
d d^{c} g_{Y, t}=\left[\mu_{Y, t}\right]-\delta_{Y}
$$

with $\mu_{Y, t}$ a smooth form on $\mathbb{C}^{2}$. The explicit given heat kernel for $X$ allows to write down $\mu_{Y, t}$ explicitly

$$
\mu_{Y, t}=\frac{-1}{(8 \pi t)^{2}} \cdot \exp \left(\frac{-\left\|z_{1}\right\|^{2}-\left\|z_{2}\right\|^{2}}{4 t}\right) d z_{1} \wedge d \bar{z}_{1} \wedge d z_{2} \wedge d \bar{z}_{2} .
$$

Resuming the computations of sections 3 and 4 we find that

$$
\begin{aligned}
g_{Y, t} & =4 \pi \Lambda \int_{0}^{t} \mu_{Y, s} d s \\
& =\frac{-1}{16 \pi} \Lambda\left(\frac{4}{\left\|z_{1}\right\|^{2}+\left\|z_{2}\right\|^{2}} \exp \left(\frac{-\left\|z_{1}\right\|^{2}-\left\|z_{2}\right\|^{2}}{4 t}\right) d z_{1} \wedge d \bar{z}_{1} \wedge d z_{2} \wedge d \bar{z}_{2}\right) \\
& =\frac{i \exp \left(\frac{-\left\|z_{1}\right\|^{2}-\left\|z_{2}\right\|^{2}}{4 t}\right)}{8 \pi\left(\left\|z_{1}\right\|^{2}+\left\|z_{2}\right\|^{2}\right)}\left(d z_{1} \wedge d \bar{z}_{1}+d z_{2} \wedge d \bar{z}_{2}\right) .
\end{aligned}
$$




\section{The spectrum of the Laplace operator on $\mathbb{P}^{1}$}

8.1 We repeat here the results of [5]. Let $X=G / K$ be a homogeneous space equipped with a $G$-invariant metric. We assume that $X$ is compact. Let $U$ be a hermitian vector space with an action of $K$ preserving the metric on $U$. We obtain a hermitian vector bundle $E:=G \times{ }_{K} U$ on $X$.

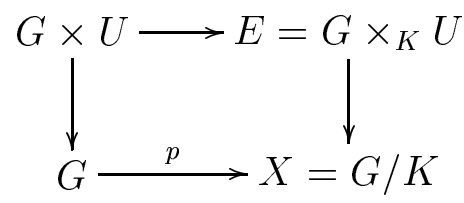

We obtain a canonical isomorphism $\mathcal{C}^{\infty}(G, U)=\mathcal{C}^{\infty}\left(G, p^{*} E\right)$. On $\mathcal{C}^{\infty}(G, U)$ we have a $G$-action given by $g \cdot \varphi(x)=\varphi\left(g^{-1} x\right)$. We have in $\mathcal{C}^{\infty}(G, U)$ the subspace of the $K$-invariant sections

$$
\mathcal{C}^{\infty}(G, K, U)=\left\{\varphi \in \mathcal{C}^{\infty}(G, U) \mid \varphi(g \cdot k)=k^{-1} \varphi(g) \text { for all } k \in K\right\},
$$

which is naturally identified with $\mathcal{C}^{\infty}(X, E)$. For an eigenvalue $\lambda$ of $\Delta$ we denote by $E_{\lambda}$ its eigenspace. Obviously, $E_{\lambda}$ is a $G$-invariant subspace of $\mathcal{C}^{\infty}(X, E)$ of finite dimension. Decomposing it into its irreducible components we obtain a further decomposition of $E_{\lambda}$. If at the other hand $V$ is an irreducible representation of $G$ with a $G$-embedding $\iota: V \rightarrow \mathcal{C}^{\infty}(G, K, U)$, then since $\Delta$ is $G$-invariant $\iota(V)$ has to be contained in an eigenspace $E_{\lambda}$, for a certain $\lambda$. Thus, we have to determine the set $\operatorname{Hom}_{G}\left(V, \mathcal{C}^{\infty}(G, K, U)\right)$ for all irreducibel representations $V$ of $G$. By Frobenius reciprocity we have

$$
\begin{aligned}
\operatorname{Hom}_{K}(V, U) & \stackrel{\sim}{\rightarrow} \operatorname{Hom}_{G}\left(V, \mathcal{C}^{\infty}(G, K, U)\right) \\
\psi & \mapsto \Psi \text { with } \Psi(v)(g)=\psi\left(g^{-1} v\right)
\end{aligned}
$$

8.2 Eigenfunctions of $\Delta$ in $\mathcal{C}^{\infty}\left(\mathbb{P}^{1}, \mathbb{C}\right)$. We apply the above to $G=\mathrm{SU}(2), K=S(\mathrm{U}(1) \times$ $\mathrm{U}(1)), X=\mathbb{P}^{1}$, and $U=\mathbb{C}$ with the trivial action of $K$. We consider the space $W=\mathbb{C}\langle x\rangle \oplus$ $\mathbb{C}\langle y\rangle$ with the natural $G$-action. It implies representations of $G$ on $V_{n}:=\operatorname{Sym}^{n}(W)$, for all integers $n \geq 0$. The representations $\left\{V_{n}\right\}_{n \geq 0}$ are a complete set of inequivalent irreducible representations of $G$.

The decomposition of $V_{n}$ into irreducible $K$-spaces is given by

$$
V_{n}=\mathbb{C}\left\langle x^{n}\right\rangle \oplus \mathbb{C}\left\langle x^{n-1} y\right\rangle \oplus \ldots \oplus \mathbb{C}\left\langle y^{n}\right\rangle
$$

where $\mathrm{U}(1) \cong K$ acts via $\left(\xi, x^{k} y^{n-k}\right) \mapsto \xi^{2 k-n} x^{k} y^{n-k}$ on $\mathbb{C}\left\langle x^{k} y^{n-k}\right\rangle$. We obtain that any $K$ homomorphism from $V_{n}$ to $U$ must send $x^{k} y^{n-k}$ to zero unless $n=2 k$. Thus, we conclude that $\operatorname{Hom}_{K}\left(V_{n}, \mathbb{C}\right)$ is zero for $n$ odd, and of dimension one for $n$ even. Hence, there exists a unique $G$-equivariant embedding $\Phi_{2 n}: V_{2 n} \rightarrow \mathcal{C}^{\infty}(G, K, U)$ up to scalars. The above remarks imply that $\Phi_{2 n}\left(V_{2 n}\right)$ is contained in an eigenspace $E_{\lambda}$ for a certain $\lambda$. Thus, a Hilbert space basis for $\mathcal{C}^{\infty}\left(\mathbb{P}^{1}, \mathbb{C}\right)$ consisting of eigenfunctions of the Laplace operator on $\mathbb{P}^{1}$ is given by the set $\left\{f_{n, k}:=\Phi_{2 n}\left(x^{k} y^{2 n-k}\right)\right\}_{n \geq 0, k=0, \ldots, 2 n}$.

8.3 Next we compute the eigenfunctions on the open subset $\mathbb{C} \cong U_{0} \subset \mathbb{P}^{1}$ with the complex coordinate $z$. Over $U_{0}$ we have a section $\sigma$ of $p$ given by

$$
\sigma(z)=\frac{1}{\sqrt{1+|z|^{2}}}\left(\begin{array}{cc}
1 & -\bar{z} \\
z & 1
\end{array}\right)
$$

The standard action of $\sigma(z)^{-1}$ on $W$ is given by

$$
\sigma(z)^{-1}(x)=\frac{1}{\sqrt{1+|z|^{2}}}(x-z y) \text { and } \sigma(z)^{-1}(y)=\frac{1}{\sqrt{1+|z|^{2}}}(\bar{z} x+y) .
$$


Eventually we can give the local form of $f_{n, k}$ in $U_{0}$

$$
\begin{aligned}
f_{n, k}(z) & =\frac{x^{n} y^{n}-\text { part of }\left(\left(\sigma(z)^{-1}(x)\right)^{k}\left(\sigma(z)^{-1}(x)\right)^{2 n-k}\right)}{\left(1+|z|^{2}\right)^{n}} \\
& =\frac{1}{(n !)^{2}\left(1+|z|^{2}\right)^{n}} \frac{\partial^{n}}{\partial x^{n}} \frac{\partial^{n}}{\partial y^{n}}\left(\left(\sigma(z)^{-1}(x)\right)^{k}\left(\sigma(z)^{-1}(x)\right)^{2 n-k}\right) \\
& =\left\{\begin{array}{c}
\frac{\bar{z}^{n-k}}{\left(1+|z|^{2}\right)^{n}} \sum_{l=0}^{k}(-1)^{k-l}\left(\begin{array}{c}
k \\
l
\end{array}\right)\left(\begin{array}{c}
2 n-k \\
n-l
\end{array}\right)|z|^{2 k-2 l} \quad, \text { for } k \leq n \\
\frac{z^{k-n}}{\left(1+|z|^{2}\right)^{n}} \sum_{l=0}^{2 n-k}(-1)^{k+n+l}\left(\begin{array}{c}
k \\
n-l
\end{array}\right)\left(\begin{array}{c}
2 n-k \\
l
\end{array}\right)|z|^{2 l}, \text { for } k \geq n .
\end{array}\right.
\end{aligned}
$$

Finally, we give the eigenfunctions for the first eigenvalues.

$$
\begin{aligned}
& f_{0,0}(z) \equiv 1 \quad f_{1,0}(z)=\frac{2 \bar{z}}{1+|z|^{2}} \quad f_{1,1}(z)=\frac{1-|z|^{2}}{1+|z|^{2}} \\
& f_{1,2}(z)=\frac{-2 z}{1+|z|^{2}} \quad f_{2,0}(z)=\frac{6 \bar{z}^{2}}{\left(1+|z|^{2}\right)^{2}} \quad f_{2,1}(z)=\frac{3 \bar{z}\left(1-|z|^{2}\right)}{\left(1+|z|^{2}\right)^{2}} \\
& f_{2,2}(z)=\frac{1-4|z|^{2}+|z|^{4}}{\left(1+|z|^{2}\right)^{2}} \quad f_{2,3}(z)=\frac{-3 z\left(1-|z|^{2}\right)}{\left(1+|z|^{2}\right)^{2}} \quad f_{2,4}(z)=\frac{6 z^{2}}{\left(1+|z|^{2}\right)^{2}} .
\end{aligned}
$$

8.4 Recalling that the Laplace operator $\Delta=d d^{*}+d^{*} d$ for functions on $U_{0}$ has the form $\Delta=-4 \pi(1+z \bar{z})^{2} \frac{\partial}{\partial z} \frac{\partial}{\partial \bar{z}}$, we easily compute that $\Delta f_{n, 0}=4 \pi n(n+1) f_{n, 0}$. Since $\Phi_{2 n}\left(V_{2 n}\right)$ is contained in an eigenspace we conclude

$$
\Delta f_{n, k}=4 \pi n(n+1) f_{n, k} \quad \text { for } k=0, \ldots 2 n .
$$

Knowing all eigenvalues of $\Delta$ with their multiplicities, we can write down the zeta function $\zeta_{\mathbb{P}_{F S}^{1}}$ of $\mathbb{P}^{1}$ with the Fubini-Study metric

$$
\zeta_{\mathbb{P}_{F S}^{1}}(s)=\sum_{n \geq 1} \frac{2 n+1}{(4 \pi n(n+1))^{s}} .
$$

8.5 The orthogonality of eigenspaces of $\Delta$ implies that the scalar product

$$
\left(f_{n, k}, f_{n^{\prime}, k^{\prime}}\right)=\int_{\mathbb{P}^{1}} f_{n, k} \overline{f_{n^{\prime}, k^{\prime}} \omega}
$$

is zero unless $n=n^{\prime}$. From our formula for the $f_{n, k}$ it follows immediately that the scalar product $\left(f_{n, k}, f_{n, k^{\prime}}\right)$ is zero, for $k \neq k^{\prime}$. Hence, the $f_{n, k}$ form an orthogonal basis. By straightforward calculations we obtain

$$
\left\|f_{n, n}\right\|^{2}=\frac{1}{2 n+1}
$$

8.6 Now we are able to derive a formula for the canonic harmonic green current of the Dirac distribution $\delta_{0}$. We set

$$
\delta_{0}=\sum_{n \geq 0, k=0 \ldots 2 n} a_{n, k} f_{n, k} \omega .
$$

Applying this distribution to the function $f_{m, l}$, we obtain

$$
f_{m, l}(0)=\int_{\mathbb{P}^{1}} \sum_{n \geq 0, k=0 \ldots 2 n} a_{n, k} f_{n, k} f_{m, l} \omega .
$$


Using the equality $f_{m, l}=(-1)^{m+l} \overline{f_{m, 2 m-l}}$, and the pairwise orthogonality of the $f_{n, k}$, this yields $f_{m, l}(0)=a_{m, 2 m-l} \int_{\mathbb{P}^{1}} f_{m, 2 m-l} f_{m, l} \omega$. Since $f_{n, k}(0)=\delta_{n, k}$, we obtain

$$
a_{m, l}=(2 m+1) \delta_{m, l} .
$$

Thus, we get for the Dirac distribution $\delta_{0}$

$$
\delta_{0}=\sum_{n \geq 0}(2 n+1) f_{n, n} \omega
$$

which allows us to write down the heat flow $\mu_{0, t}$ of $\delta_{0}$

$$
\mu_{0, t}=\sum_{n \geq 0}(2 n+1) \exp \left(\frac{-t}{4 \pi n(n+1)}\right) f_{n, n} \omega .
$$

We deduce that the Green operator $G(0)$ of $\delta_{0}$ is given by

$$
G(0)=\sum_{n>0} \frac{(2 n+1) f_{n, n}}{4 \pi n(n+1)} \omega
$$

and that the canonical harmonic Green current has the form

$$
g_{0}=\sum_{n>0} \frac{(2 n+1) f_{n, n}}{n(n+1)} .
$$

The uniqueness of the canonical harmonic Green current and 7.1 thus implies

$$
\log \left(1+\frac{1}{z \bar{z}}\right)-1=\sum_{n>0} \sum_{l=0}^{n} \frac{(2 n+1)(-1)^{l}\left(\begin{array}{c}
n \\
l
\end{array}\right)^{2}\|z\|^{2 l}}{n(n+1)\left(1+\|z\|^{2}\right)^{n}} .
$$

\section{$9 \quad$ Further questions}

9.1 Levine constructs in [7] Green currents for linear subspaces of the projective space $\mathbb{P}^{n}$. In the case of a divisor these currents coincide up to a constant with the canonical Green current because of lemma 4.5. The example 7.1 shows that this constant is not zero. However, for linear subspaces of higher codimension these two currents differ considerably as we will see in a moment.

9.2 Let $P$ be a point in the projective plane $\mathbb{P}^{2}$. Let $P$ locally be given by $\left(z_{1}=z_{2}=0\right)$. The canonical harmonic Green current $g_{P}$ is locally given by

$$
g_{p}=\frac{i}{2 \pi\left(\left|z_{1}\right|^{2}+\left|z_{2}\right|^{2}\right)}\left(d z_{1} \wedge d \bar{z}_{1}+d z_{2} \wedge d \bar{z}_{2}\right)+\alpha
$$

where $\alpha$ is some smooth form (see 4.7).

Let $\mathbb{P}_{P}^{2}$ be the blow up of $\mathbb{P}^{2}$ in the point $P$, and $f: \mathbb{P}_{P}^{2} \rightarrow \mathbb{P}^{2}$ the natural projection. As usual the exceptional divisor $f^{-1}(P)$ is denoted by $E$. Locally around $P$ the space $\mathbb{P}_{P}^{2}$ is embedded into $\mathbb{P}^{2} \times \mathbb{P}^{1}$ and given by the equality $z_{1} Z_{2}=z_{2} Z_{1}$ where $\left(Z_{1}: Z_{2}\right)$ are the homogeneous coordinates on $\mathbb{P}^{1}$. Considering the open subset where $Z_{1} \neq 0$, we obtain local coordinates $\left(z_{1}, Z_{2}\right)$ on $\mathbb{P}_{P}^{2}$. The exceptional divisor is given by $z_{1}=0$. In these coordinates we compute the pull back $f^{*} g_{P}$ to be (up to some smooth form)

$$
f^{*} g_{P}=\frac{i}{2 \pi}\left(\frac{d z_{1} \wedge d \bar{z}_{1}}{\left|z_{1}\right|^{2}}+\frac{d Z_{2} \wedge d \bar{Z}_{2}}{1+\left|Z_{2}\right|^{2}}+Z_{2} \frac{d z_{1}}{z_{1}} \wedge \frac{d \bar{Z}_{2}}{1+\left|Z_{2}\right|^{2}}+\bar{Z}_{2} \frac{d \bar{z}_{1}}{\bar{z}_{1}} \wedge \frac{d Z_{2}}{1+\left|Z_{2}\right|^{2}}\right)
$$


Thus we see that $f^{*} g_{P}$ is no $L^{1}$-form. Therefore $f^{*} g_{P}$ is not of logarithmic type in the sense of [2]. In [3] Gillet and Soulé show that Levine's form is of logarithmic type. Thus, the canonical Green current differs from Levine's current.

9.3 Analogous computations show that the canonical Green current of a cycle $Y$ in $X$ is not of logarithmic type, unless $Y$ is a divisor. The reason why our canonically defined object does not fit into the concept of currents of logarithmic type might be that to construct currents of logarithmic type we are considering not only the embedding $\iota: Y \rightarrow X$ but the blow up diagram

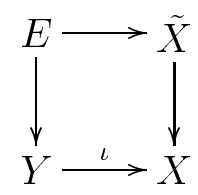

which plays a central role in intersection theory.

9.4 Having a canonical current we could hope to simplify the intersection theory. However, the *-product of canonical Green currents $g_{Y}$ and $g_{Z}$ gives a Green current for the intersection product $Y . Z$ but not the canonical one. There are various reasons for this, for example: the *-product $g_{Y} * g_{Z}$ is singular along $Y$ whereas $g_{Z} * g_{Y}$ is singular along $Z$. Yet, the difference $g_{Y} * g_{Z}-g_{Z} * g_{Y}$ is an exact current (see [2]). Another reason is that the wedge product of harmonic forms is not harmonic.

\section{References}

[1] Berline, N.; Getzler, E.; Vergne, M., Heat Kernel and Dirac Operators, Grundlehren der math. Wiss. 298, Springer, Berlin, 1996.

[2] Gillet, H.; Soulé, C., Arithmetic intersection theory, IHES 72 (1990), 93-174.

[3] Gillet, H.; Soulé, C., Characteristic classes for algebraic vector bundles with Hermitian metrics, II, Annals of Math. 131 (1990), 205-238.

[4] Griffith, P.; Harris, J., Principles of algebraic geometry, Pure and Applied Mathematics, John Wiley \& Sons, New York, 1978.

[5] Ikeda, A. and Taniguchi, Y., Spectra and Eigenforms of the Laplacian on $S^{n}$ and $\mathbb{P}^{n}(\mathbb{C})$, Osaka J. Math. 15 (1978), 515-546.

[6] Lang, S., Introduction to Arakelov theory, Springer-Verlag, New York, 1988.

[7] Levine, H., A theorem on holomorphic mappings into complex projective space, Ann. of Math. 71 (1960), 529-535.

[8] Soulé, C.; Abramovich, D.; Burnol J.-F.; Kramer, J., Lectures on Arakelov theory, Cambridge Univ. Press, Cambridge, 1992.

[9] Wells, R. O. jun., Differential analyis on complex manifolds, Springer-Verlag, New York, 1980. 